\title{
Mediaciones Algorítmicas para la Percepción de la Ciudad y su Apropiación Agonista
}

\section{Algorithmic mediations for the perception of the city, and its agonistic appropriation}

\author{
Diego Gómez \\ Universidad de Chile, Chile \\ diegogomez@uchilefau.cl \\ Mario Marchant \\ Universidad de Chile, Chile \\ mmarchant@uchilefau.cl \\ Loreto Ulloa \\ Universidad de Chile, Chile \\ loreto.ulloa.i@gmail.com \\ Bárbara Echaíz \\ Universidad de Chile, Chile \\ b.echaiz.b@gmail.com
}

\author{
Carla Ponzano \\ Universidad de Chile, Chile \\ nisim2510@gmail.com \\ Rocío Hurtado \\ Universidad de Chile, Chile \\ hurtadorobado@gmail.com
}

\author{
Adolfo Álvarez \\ Universidad de Chile, Chile \\ alvarezdumont@gmail.com
}

\begin{abstract}
This paper presents conceptual and theoretical basis, methodological processes, and ongoing developments for the research work done within the Design and Agonism group at the Department of Design of the School of Architecture and Urbanism of the University of Chile. The research problem guiding this work has been defined as algorithmic mediations for the perception of the city, as vehicles for agonistic citizen appropriation in a hypothetic Santiago, Chile.
\end{abstract}

Keywords: Adversarial design; Agonism; Mediations; Citizenship; Media Arts.

\section{Introducción}

A través de este artículo buscamos presentar las bases y desarrollo del trabajo de investigación en curso que realizamos desde el grupo Diseño y Agonismo del Departamento de Diseño de la Facultad de Arquitectura y Urbanismo de la Universidad de Chile; cuyo problema de estudio hemos definido como mediaciones algorítmicas para la percepción de la ciudad, como vehículo de apropiación ciudadana agonista, en un Santiago de Chile hipotético.

Originalmente, el área de referencia que nos permitió elaborar esta idea de investigación, se ubicó en la intersección entre lo que Carl Disalvo ha llamado diseño adversario (Disalvo, 2012), reflexiones sobre estética y percepción planteadas por Paul Virilio y el campo de las artes mediales; en la cual vimos terreno fértil para la investigación creativa - con foco especulativo - para disciplinas como el diseño y la arquitectura. Desde allí, hemos podido expandir nuestro modelo conceptual levantando un proyecto que actualmente cuenta con el financiamiento de un fondo interno de la Facultad de la que somos parte, y un Fondo de Cultura del Consejo Nacional de la Cultura y las Artes.
Con todo, nos preguntamos si la acción de la percepción, es decir el percibir, constituye un acto estético, y desde ahí, también un acto político. Más aún, nos preguntamos si es posible mediar con tecnología en aquellos actos, y particularmente, si es posible diseñar mediaciones de carácter algorítmico que lleven esas relaciones estético-políticas a un espacio de disenso y confrontación que potencie la apropiación ciudadana. Así, hemos ido realizando nuestro trabajo entorno a mediaciones algorítmicas para la percepción de la ciudad, entendidas éstas como nuestro objeto de estudio y, que luego de la fase de campo en la que nos encontramos hoy, nos llevará al diseño y construcción de prototipos que asuman de manera consistente el rol de tales objetos, para lo cual exploraremos perspectivas de public sensing y técnicas de physical computing. Ahora bien, un acento fundamental para este itinerario investigativo, recae en otorgarle al proceso completo un espesor de pensamiento sustantivo que nos permita validar la creación en diseño y arquitectura como recurso propio para la investigación en estas áreas, sumándonos a lo que en otros contextos se ha denominado practice-led research. 
En lo que sigue, buscaremos por una parte dejar en claro el marco teórico-conceptual que nos ha permitido abordar nuestro problema de investigación del modo en que lo estamos haciendo, y justificar por qué es relevante tratar este tema desde el diseño hoy. Por otro lado, intentaremos dar cuenta de la estructura metodológica que guía nuestro proceso, haciendo foco en las innovaciones que lo fundan, cuáles son sus sustentos bibliográficos, y cuáles son sus proyecciones y potencialidades para nuestras disciplinas.

\section{Bases teóricas y conceptuales}

Una vez introducido nuestro problema de investigación, creemos necesario profundizar en los conceptos que lo caracterizan. Éstos son básicamente tres. A saber; la cuestión de la mediación, lo algorítmico y el concepto de agonismo. Comenzaremos con los dos primeros, pues si bien son altamente relevantes para nuestro trabajo, son más sencillos y probablemente también familiares para el lector. Cerraremos este punto entonces, profundizando en el tercer elemento, pues nos atrevemos a decir que es el concepto central de nuestro trabajo $y$, el que finalmente le otorga un discurso y guión; siendo probablemente, el más ajeno al lenguaje que rodea nuestras especialidades.

\section{La cuestión de la mediación}

Para abordar este punto, invariablemente debemos comenzar por acercarnos a la interfaz y las relaciones teórico-prácticas que la rodean. Con ese fin, es que nos permitimos mencionar aquí algunas de las ideas expuestas por Eduardo Sabrovsky en el texto El Concepto de Interfaz: Notas sobre ciencia de lo artificial, arquitectura y biopoder (Sabrovsky, 2012). Allí, el autor se inspira en el libro de Herbert Simon, The Sciences of the Artificial, para señalar que las disciplinas del diseño corresponden precisamente a lo que denominará ciencias de lo artificial, es decir, a las prácticas ocupadas de lo que debe ser en contraste al lo que es que ocupa a las Ilamadas ciencias duras. En esta perspectiva, para las ciencias de lo artificial la interfaz será el borde que sirve de puente entre un medio ambiente exterior y uno interior (Sabrovsky, 2012). Este enfoque cientificista que finalmente subordina estas ciencias de lo artificial a los paradigmas propios de las ciencias naturales, es el que ha regido el concepto de interfaz, representado finalmente a través de artefactos, es decir, de artificios.

En otra línea, surge la cuestión de la mediación, lo cual más que una construcción artificial, es decir, un artefacto - como sí lo sería la interfaz - es un espacio de acción subjetivo y por lo mismo, abierto a la incertidumbre. Así, podemos decir que al hablar de mediaciones buscamos alejarnos de áreas absolutamente subordinadas a los paradigmas naturalistas, y más bien buscamos instalarnos en los dominios subjetivos propios de las ciencias sociales, y más particularmente, en las humanidades. Con este cambio de foco, lo que queremos es instalar la mirada del sujeto como foco principal de la cuestión y, entendiendo que éste conoce a través de la interpretación, es decir de la subjetividad, validar lo incierto como condición propia de la mediación. Ahora bien, estas mediaciones pueden ser sin duda cosas artificiales, aunque a veces también podrán ser cosas naturales e incluso individuos humanos. De este modo, para entender estas vinculaciones nos acercamos al trabajo de Bruno Latour y la Teoría del Actor Red (TAR), la que nos lleva a hablar de actores relacionados por asociaciones en las cuales algunos actores serán mediadores (Latour, 2008). Entenderemos así entonces la cuestión de la mediación, pues nos permite abordar el problema de investigación poniendo en diálogo de modo balanceado a sujetos, aparatos mediadores, estructuras políticas y ciudad.

\section{Lo algorítmico}

Como probablemente el lector entiende, un algoritmo es una lista finita de instrucciones lógicas que constituyen un procedimiento para realizar un cálculo o resolver un problema concreto. Estos aparatos lógicos, propios de las matemáticas y ciencias de la computación, están presentes hoy de manera ubicua en las sociedades contemporáneas. Un amplio rango de artefactos (semáforos, automóviles, trenes subterráneos, cámaras de vigilancia, imágenes medializadas, sistemas de iluminación, puertas de edificios, sistemas de pago electrónicos, teléfonos celulares, aplicaciones móviles y por cierto, computadores), los contienen y necesitan para su funcionamiento, permeando y condicionando nuestras vidas. Es precisamente allí donde radica lo que llamamos lo algorítmico, entendido como el problema de la condicionalidad que los medios y la tecnología impone en los modos de vida de los individuos hoy.

No es posible entender las mediaciones que nos interesa confrontar, ni menos abordarlas de manera consistente - para luego diseñarlas - si no existe una atención proporcionada a las complejidades que lo algorítmico arroja a los modos cómo los individuos - de modo condicionado - perciben, conocen y se desenvuelven en las ciudades contemporáneas. Tal vez nos deberíamos preguntar ya, si las personas han venido siendo entrenadas para actuar de manera condicionada, incluso cuando no media en su accionar artefacto concretamente algorítmico alguno, sino más bien que ha sido lo algorítmico en sí, lo que se ha instalado en los modos de accionar de los individuos.

\section{El concepto de agonismo}

Como señalamos anteriormente, éste es el elemento conceptual que mayor relevancia tiene en nuestro problema de estudio, otorgándole un guión y discurso a nuestro trabajo de investigación. Agonismo es un término acuñado por la cientista política belga, Chantal Mouffe, y con el cual buscar dar cuenta de la importancia que tendría para el cultivo de democracias saludables y pluralistas los espacios de disenso y confrontación. 
Para Mouffe una de las mayores razones de la crisis que enfrentan los sistemas democráticos modernos, radica precisamente en la negación de los espacios de conflicto, los que habrían sido reemplazados por la constante búsqueda del consenso, imponiendo así lo que ella denomina el Zeitgeist pospolítico (Mouffe, 2007). La mirada de Mouffe se aleja de esta perspectiva, y para desarrollar el marco conceptual que le permita desplegar su pensamiento, se ha acercado, a pesar de ser una intelectual de izquierda, a las ideas del alemán Carl Schmitt; filósofo y político fascista. Ha interesado a Mouffe la idea de antagonismo de Schmitt, la cual se basa en el entendido que en política deben existir sólo relaciones amigo - enemigo, donde se debe buscar precisamente destruir al antagonista. Si bien Mouffe es contraria a tal extremismo, sí valora el rol que tiene el enfrentamiento en la teoría política del mencionado filósofo alemán. Así, Mouffe ha buscado desarrollar los planteamientos teóricos que permitan levantar una opción de democracia contemporánea que sí considere los espacios de confrontación, ya que según ella, la democracia tiene una naturaleza conflictual inherente. Ahora bien, para Mouffe, esta visión de democracia - a la cual añade el calificativo de radical-pluralista - se basa en la relación entre adversarios (y no enemigos), los cuales requieren de espacios instalados para desplegar sus discursos. A estas acciones de confrontación y disenso en dichos espacios, es lo que Mouffe ha llamado agonismo.

Ahora, entendemos si el lector en este punto ha comenzado a cuestionar cuál es el lugar de las disciplinas del diseño en todo esto. Pues bien, diremos que precisamente ha sido desde allí que nos hemos involucrado con estos conceptos. En su libro Adversarial Design, el académico y diseñador estadounidense Carl DiSalvo, nos habla del papel que las cosas diseñadas pueden tener en un contexto donde el agonismo es un tipo de actividad ciudadana que se busca rescatar y desarrollar de modo activo (DiSalvo, 2012). Así, DiSalvo acuña el concepto diseño adversario refiriéndose precisamente a esas cosas que han sido diseñadas para propiciar el accionar agonista (DiSalvo, 2012). Más aún, el autor hace énfasis en el potencial particular que tendría la computación - nosotros hemos preferido el término algorítmico - como medio para el diseño adversario, dado su ubicuidad en la cultura contemporánea.

De este modo, probablemente comienza a ser visible para el lector el marco conceptual que nos permite sustentar, y al mismo tiempo desarrollar, el problema de estudio. Nuestro interés por investigar sobre el diseño de mediaciones algorítmicas para la percepción, que vehiculicen apropiaciones ciudadanas agonistas implica preguntarse cuáles son las características que deberán tener tales mediaciones, para que efectivamente propicien acciones de disenso y confrontación, a la vez que permiten vincularse a la ciudad. En lo que sigue, intentaremos dar cuenta de cómo estamos llevando a cabo esta tarea, entiendo que es un proceso en curso, el cual aún tiene por delante alrededor de dos tercios de sus fases de desarrollo.

\section{Estructura Metodológica}

En la práctica, nuestro proyecto de investigación consta de siete fases: revisión bibliográfica, rastreo de muestra, trabajo de campo inicial, diseño y prototipado, iteraciones, refinamiento de prototipos y sistematización. De éstas, ya hemos llevado a cabo las dos primeras, estamos finalizando la tercera y a mediados de octubre de 2013, si todo avanza según lo planeado, estaremos ingresando a la fase de diseño y prototipado. A continuación presentamos algunos aspectos de las acciones ya realizadas.

\section{Revisión bibliográfica}

Esta etapa se inició cuando ya se contaba con la certeza de dos fondos de investigación adjudicados (FAU-UCH y FONDART), y para cuyas postulaciones se habían realizado consultas bibliográficas iniciales, específicamente a los textos Adversarial Design de Carl DiSalvo y Estética de la Desaparición de Paul Virilio. A partir de ahí nuestro trabajo consistió en profundizar en el análisis de aquellas publicaciones, lo cual nos llevó a expandir la revisión. Así DiSalvo nos dirigió hacia Chantal Mouffe; de quien posteriormente hemos consultado En torno a lo político; y a estudiar el texto Hertzian Tales del diseñador y académico inglés, Anthony Dunne. Esto, sumado al libro de Virilio, nos acercó también a Bruno Latour, de quien estudiamos Reensamblar lo social para introducirnos en la teoría del actor-red.

En base a lo anterior, los estudiantes integrantes del equipo investigador desarrollaron artículos breves, centrándose cada uno en conceptos específicos que las lecturas bases hicieron emerger. Este ejercicio permitió la realización de un primer paper, el cual fue posteriormente presentado en el marco del II Encuentro Nacional de Nuevos Medios. Todo este material se encuentra disponible en el sitio web del grupo de investigación ${ }^{1}$, obedeciendo a la política de difusión abierta que nos anima.

\section{Rastreo de muestra}

Particularmente influenciados por las ideas de Bruno Latour, decidimos no definir una muestra para nuestro trabajo de campo, como usualmente lo señala la norma metodológica para la investigación académica. Por el contrario, quisimos alejarnos de este procedimiento en pos de avanzar hacia rastrear nuestra muestra. Para tales efectos, diseñamos una actividad que nos permitiera observar cómo las personas se vinculan a otros actores en un contexto de disenso y confrontación, es decir, realizar un rastreo de asociaciones para el agonismo.

Dicha actividad consistió en montar una sencilla instalación en distintos puntos de la ciudad, a través de la cual las personas podían manifestar, con distintos grados de exposición, sus opiniones respecto a personajes, organizaciones y problemas de índole política y/o actualidad. Estas opciones se presentaban a

\footnotetext{
${ }^{1}$ http://disenoyagonismo.uchilefau.cl
} 
través de papeletas las cuales podían recibir anotaciones, que posteriormente podían pegarse en un panel, depositarse en una urna, o bien, utilizar un megáfono para señalar lo que en ellas se había escrito. Luego de aquello, las personas que participaron de la actividad fueron abordadas, para saber si estaban interesadas en participar de nuestra investigación. A partir de aquello se confeccionó una base de datos que fue creciendo gracias al complemento dado por carteles que se pegaron en los mismos puntos de la ciudad donde la instalación había sido montada, y que también se difundieron a través de redes sociales. Finalmente, parte de las personas en esa base de datos fueron invitadas a reuniones informativas sobre los procesos y alcances de la investigación, donde tomaban la decisión de participar o no en ella. De esta manera, nueve personas decidieron participar activamente de la investigación, lo que nos permitió avanzar hacia la fase de trabajo de campo.

\section{Trabajo de campo inicial}

El trabajo de campo correspondió principalmente a actividades de etnografía, las cuales tomaron forma de acompañamientos a los individuos estudiados, en diferentes contextos (laboral, ocio, transporte y personal). A lo largo de este proceso nos hemos concentrado en observar los vínculos que estas personas tienen con tres aspectos, a saber, su relaciones con la tecnología, con la manifestación de desacuerdos políticos y con su ciudad.

Así al observar sus relaciones con la tecnología nos interesó conocer sin duda, cuáles son los aparatos que usan, pero también, y quizá sobre todo, cómo son estos usos y si existe reflexión al respecto de parte de los mismos sujetos; además de enterarnos sobre los alcances que tiene la categoría tecnología para ellos. Por otro lado, en su relación con desacuerdos políticos, buscamos saber hasta que punto están dispuestos a manifestarlos, si realizan reflexión respecto a esas manifestaciones y cómo perciben la manifestaciones de otros al respecto. Finalmente, en su vínculo con la ciudad quisimos conocer cómo la viven, esto es, los modos en que la recorren, que lugares visitan y qué hacen en cada uno de ellos, pero por sobre todo, nos interesó saber si la perciben de modo consciente y qué significado tenía esa acción para ellos.

Todos estos datos se han ido documentando a través de notas, fotografías, vídeos y registro de audio. Hacia inicios de octubre de 2013 esta información se comenzó a sistematizar en pos de establecer áreas con mayor potencial de desarrollo conceptual y también acotar el grupo de personas con las cuales estamos trabajando, con miras de avanzar hacia la siguiente fase, donde diseñaremos un prototipo para cada individuo.

\section{Continuación y Proyecciones}

El siguiente paso en nuestro proyecto es ingresar en la fase de diseño y prototipado. Para eso, comenzaremos con el desarrollo conceptual de los prototipos que buscamos construir, y que estimamos serán alrededor de cinco. En aquel punto, será fundamental el input que nos otorgue la sistematización de información proveniente del trabajo etnográfico. A la vez, ubicaremos estos conceptos dentro de escenarios hipotéticos para la ciudad de Santiago, pues uno de nuestros objetivos es precisamente poner a prueba los alcances que acciones de creación especulativa puedan tener para la investigación-de basepráctica en disciplinas del diseño.

Desde ahí, avanzaremos hacia la construcción misma de dichos prototipos. Con este fin, trabajaremos con técnicas de physical computing en la puesta en marcha de dispositivos funcionales pero no acabados en sus terminaciones. Con esto buscamos enfocarnos en el potencial para el public sensing, la interactividad y la condición wearable que nos ofrecen dichas técnicas, pues, vemos ahí interesantes oportunidades para abordar nuestro problema de estudio con modelos concretos. Al mismo tiempo, queremos beneficiarnos de la flexibilidad para la realización de pruebas e iteraciones que presentaría dicha aproximación, pues estos prototipos no tendrían realmente sentido sin una relación performática situada y en vínculo con los sujetos para quienes se diseñarán.

No será sino hasta saldados los aspectos anteriores que nos moveremos hacia la refinación de estos prototipos, centrándonos allí en sus terminaciones formales y aspectos de fabricación. Éste y los procesos anteriores, serán documentados con fotografías y video con miras a la fase de sistematización, la cual, además del levantamiento de conclusiones conceptuales y de procedimiento, contendrá recursos técnicos sobre el diseño y construcción de los prototipos, pues nos interesa compartir libremente los conocimientos desarrollados en las distintas fases del proyecto. Todo este material será distribuido de manera online e impresa, centrándonos en escuelas de diseño, arquitectura, arte y bibliotecas públicas.

Nuestras expectativas apuntan a, que el proceso mismo y los resultados de nuestra investigación permitan por una parte, diluir los límites de las especialidades de las disciplinas del diseño que hoy predominan en los centros universitarios chilenos, léase, diseño gráfico, diseño industrial y arquitectura. Esto, debido a que creemos firmemente en que lo medial ofrece espacios para la confluencia disciplinar y abordaje de problemas de alta relevancia para la cultura contemporánea. Al mimo tiempo, esperamos que nuestro trabajo nos permita movilizar la convicción de que el diseño con mirada pública obliga a entender la disciplina como un aparato para la divulgación de políticas, cuestión que debería constituir un problema fundamental para profesionales $y$ académicos del área. Finalmente, también buscamos que esta investigación contribuya a validar la investigación-de-basepráctica, es decir, aquella que considera las acciones de creación como procedimiento propio del hacer académico en las disciplinas del diseño. 


\section{Referencias}

DiSalvo, C. (2012). Adversarial Design. Cambridge, MA: MIT Press.

Sabrovsky, E (2012). El Concepto de Interfaz. Notas sobre ciencias de lo artificial, arquitectura y biopoder.

En A. Castillo y C. Gómez-Moya (Ed.) Arte, archivo y tecnología (pp. 75-90). Santiago, Chile: Ediciones Universidad Finis Terrae.

Virilio, P. (1988). Estética de la Desaparición. Barcelona: Anagrama.
Dunne, A. (2008). Hertzian Tales: Electronic products, aesthetic experience, and critical design. Cambridge, MA: MIT Press.

Latour, B. (2008). Reensamblar lo Social: Una introducción a la teoría del actor-red. Buenos Aires: Manantial.

Mouffe, C. (2007). En Torno a lo Político. Buenos Aires: Fondo de Cultura Económica. 